\title{
Buchkritik
}

Marcus Düwell*

\section{Wird philosophische Anthropologie als strenge Wissenschaft auftreten können?}

\author{
https://doi.org/10.1515/dzph-2018-0051
}

Thorsten Streubel. Kritik der philosophischen Vernunft. Die Frage nach dem Menschen und die Methode der Philosophie. Versuch einer methodologischen Grundlegung. Wiesbaden: Springer VS 2016, 374 S.

Die ,Frage nach dem Menschen‘ genießt seit einiger Zeit Aufmerksamkeit von verschiedenen Seiten. Insofern ist es erfreulich, dass Thorsten Streubel jetzt versucht, die philosophische Anthropologie aus der Perspektive der Phänomenologie näher zu profilieren. Sein Buch versteht sich explizit als Kritik am Naturalismus. Die gegenwärtige Situation erinnere an die umfassende zeitdiagnostische Kritik, die Husserl in seinem ,Krisis'-Buch vorgelegt hat. ${ }^{1}$ In der Linie von Husserls damaliger Kritik unternimmt Streubel den Versuch aufzuzeigen, dass nur eine als Wissenschaft entwickelte philosophische Anthropologie auf phänomenologischer Basis geeignet sei, ein angemessenes Verständnis des Verhältnisses von Geist und Gehirn zu entwickeln, das zugleich Grundlage für ethische Orientierung bieten könne (19). Ziel des Buches ist es, die Grundlegung für eine solche phänomenologische Anthropologie zu legen. Streubels Buch kann man als eine Auseinandersetzung mit Husserl lesen, die sich darum bemüht, dessen zentrale philosophische Überlegungen für ein Verständnis der Gegenwartssituation fruchtbar zu machen. Methodisch geht es also weder um Exegese noch um eine explizite Rekonstruktion, sondern vielmehr um ein Philosophieren auf der Basis der grundlegenden Annahmen Husserls, wobei bisweilen im Text und weitaus häufiger in den Fußnoten - eine explizite Auseinandersetzung mit Husserl erfolgt; weitere regelmäßige Gesprächspartner sind Immanuel Kant, Maurice Merleau-Ponty und Hermann Schmitz.

1 E. Husserl, Die Krisis der europäischen Wissenschaften und die transzendentale Phänomenologie. Eine Einleitung in die phänomenologische Philosophie (hg. v. W. Biemel), Den Haag 1962.

Kontakt: Marcus Düwell, m.duwell@uu.nl 
Teil A, „Die Frage nach dem Menschen und die Methode der Philosophie“, skizziert das Problem des Naturalismus im Hinblick auf das „Gehirn-Geist-Problem“ (9-17) sowie das Programm der „Idee strenger Wissenschaft“ (17-24). Den Hauptteil des Buches bildet Teil B, der das Programm in drei Kapiteln ausarbeitet (1. Kritik am Naturalismus, 2. Methodische Grundlegung, 3. Inhaltliche Skizze der Fundamentalanthropologie), gefolgt durch einen sehr knappen Ausblick in Teil C (361-365). Teil B beginnt im ersten Kapitel mit der kritischen Skizze des Naturalismus, die sich auf Diskussionen um das Verhältnis von Geist und Gehirn bezieht, wobei das Buch Das Gehirn und seine Wirklichkeit von Gerhard Roth ${ }^{2}$ (37-49) primärer Gegenstand der Untersuchung ist. Die kritische Besprechung dient dazu, die Aporien des Naturalismus aufzuzeigen und davon ausgehend die naturalistische Basis der analytischen Philosophie des Geistes insgesamt zu kritisieren.

Das zweite Kapitel (51-221) bietet die methodische Grundlegung des Buches, wobei Streubel im Hinblick auf den cartesianischen Zweifel in der Aktualität des Bewusstseins den archimedischen Punkt ausmacht: „Bewusstsein oder Anschauung als unmittelbare Präsenz von Präsentem ist als aktuelles Faktum absolut zweifelsresistent“ (62). Alle Erkenntnis ist gebunden an den ,augenblicklichen Strom des Erlebens“ (63). Von dort ausgehend werden Wahrheitsbegriff, Urteil, Sprachlichkeit und Historizität von Erkenntnis diskutiert und - strikt phänomenologisch - die Gebundenheit von Erkenntnisakten an die vorprädikative Erfahrung herausgearbeitet (90-95). Aufgrund der notwendigen Priorität der Anschauung und der Betonung der stets aktuellen Form des Bewusstseins ist auch jede Form von wissenschaftlicher Erkenntnis daran gebunden, und dies gilt auch für die Philosophie: „Die Erkenntnis der unbezweifelbaren Faktizität ist dabei selbst anschaulich. Die Erkenntnis übersteigt daher nicht die Anschauung, sondern setzt sie stets voraus. Denken und Erkennen sind nur deshalb für uns überhaupt etwas, weil sie sich anschaulich bekunden“ (200). Philosophie ist insofern als Selbsterkenntnis zu konzeptualisieren, wobei die Frage „Was ist der Mensch?“ nur dann eine genuin philosophische Frage ist, wenn sie das Bemühen darstellt, wesentliche Aspekte menschlichen Daseins aus diesem Grundfaktum der aktuellen Anschauung zu explizieren: Damit ist die Aufgabe formuliert, der sich die philosophische Anthropologie gegenübersieht.

Das dritte Kapitel (223-365) skizziert die inhaltlichen „Grundstücke einer Fundamentalanthropologie“ anhand des anthropologischen Sextetts von Bewusstsein, Leib, Körper, Welt, Ich und Geistigkeit. Es sei nur kurz auf einige Besonderheiten verwiesen, etwa die Konzeptualisierung des Leibes: „Der welthaltige

2 G. Roth, Das Gehirn und seine Wirklichkeit. Kognitive Neurobiologie und ihre philosophischen Konsequenzen. Frankfurt am Main 1994. 
Leib ist [...] die Einheit von fundierendem Leib und fundierter Umwelt“ (263), womit Streubel sich von der Leibkonzeption bei Merleau-Ponty und Schmitz abgrenzt. Mit der Spannung von Leib und Körper wird auch der Spracherwerb ein wesentliches Thema, denn ,eine gemeinsame Sprache und damit eine gemeinsame Gegenstands- und Kulturwelt [...] wären undenkbar, wenn sich leibliche Subjekte nicht als Körper in einer Welt wahrnehmend begegnen und die Welt zwar perspektivisch abweichend, aber ähnlich, sinnlich repräsentieren könnten“ (277, Hervorh. im Orig.). Streubel diskutiert die Natur der Fremderfahrung und die Transzendenz des Anderen: „Der Andere wird als jemand erfahren, der nicht in seinem Erscheinen aufgeht, sondern sich hierdurch nur bekundet. [...] der Andere wird mir [...] nie völlig durchsichtig und transparent“ (291). Auf der Basis dieser anthropologischen Grundbegriffe wird dann ein „eidetisches Forschungsprogramm“ skizziert (331 ff.), gewissermaßen als Antwort auf die eingangs kritisierte Tendenz des Naturalismus.

Das Buch ist als Rekonstruktion Husserl'schen Denkens ausgesprochen gelungen. Systematisches Denken auf den Spuren eines großen Philosophen ist vielleicht überhaupt ein philosophisches Genre, dessen Bedeutung unterschätzt wird. Wenn man verhindern will, dass unser Verhältnis zur philosophischen Tradition ein lediglich ,antiquarisches' (Nietzsche) ist, so muss es auch ein legitimes philosophisches Genre sein, sich auf die philosophische Tradition zu beziehen, ohne sich direkt in die Vielzahl exegetischer Details zu verlieren (womit die Notwendigkeit exegetischer Interpretationen in keiner Weise in Abrede gestellt sein soll). Streubels Überlegungen zum Leib-Körper-Verhältnis, zur Zeitlichkeit des Bewusstseins, zur Intersubjektivität, zu Räumlichkeit und vielem anderen sind ausgesprochen instruktiv. Auch scheut er nicht explizite Kritik an und Revision von zentralen Thesen Husserls, wie etwa im Abschnitt über die Überflüssigkeit der transzendentalen Reduktion (335-342). Ebenso erhellend sind zahlreiche Diskussionen zum Verhältnis des eigenen Ansatzes zu Merleau-Ponty und Schmitz, die systematisch hilfreich sind, um zu verstehen, welche systematischen Perspektiven man im Rahmen einer solchen phänomenologischen Anthropologie überhaupt entwickeln kann. Was immer man im Einzelnen von Streubels Position halten mag: Das Buch bietet eine sehr interessante und gute philosophische Diskussion. Zudem ist es durchaus lesbar und vermeidet überflüssigen Jargon - eine Tugend, die in phänomenologischen Kreisen gerne Nachahmung finden könnte.

Etwas ganz anderes ist jedoch sein Versuch, eine philosophische Anthropologie zu begründen oder zumindest ihre Grundlagen zu skizzieren. Die Skizze ist in vieler Hinsicht in der vorliegenden Form nicht überzeugend; ich erwähne nur zwei wesentliche Defizite. Die Konsequenzen des hier skizzierten Programms für die Hirnforschung bleiben ausgesprochen undeutlich. Dies ist ein gravierendes Defizit, weil dies der Einsatz des Buches war, der die ganze Untersuchung moti- 
vierte. Zunächst ist mir nicht wirklich deutlich, warum Streubel behauptet: „Das Ich wird in der Welt nur durch das Gehirn repräsentiert“ (315, Hervorh. im Orig.) statt es als ein (wenngleich zentrales) Organ aufzufassen und die Repräsentation des Ich durch den Körper in einem umfassenderen Sinne zu denken. Aber wie dem auch sei: Man wüsste gerne, wie sich aus der hier entwickelten Konzeption ein Weg zur Beurteilung dessen, was in der Hirnforschung passiert, ergibt. Auch verstünde man eigentlich gerne in Umrissen, wie sich überhaupt aus dem skizierten Programm Naturphilosophie und naturwissenschaftliche Erkenntnis verstehen lässt. Streubel entwickelt hier jedoch kein konstruktives Programm. Stattdessen wird recht breit und pauschal mit der analytischen Philosophie des Geistes abgerechnet, die samt und sonders des Naturalismus beschuldigt wird - nicht nur einzelne Positionen innerhalb der Debatte, sondern der gesamte Diskurs (342-357), der im letzten Absatz des Buches schlechthin zu schlechter Philosophie erklärt wird (365): „Die Hauptrichtungen der analytischen Philosophie des Geistes sind in diesem Sinne allesamt naturalistisch ausgerichtet [...] Wir kritisieren also nicht diese oder jenes Theorem jener Positionen. Wir kritisieren ihre spekulative Grundvoraussetzung“ (349, Hervorh. im Orig.). Um diese Behauptung zu stützen, müsste man etwas mehr tun, als ein paar Texte von Bieri, Metzinger und Roth heranzuziehen. Anders als derartige undifferenzierte Rundumschläge scheint mir die Kritik, dass dieser Diskurs die Herausforderung der kantischhusserlschen Position nicht ernst nehme (353), völlig berechtigt (wenngleich mir das Attribut des transzendentalen Idealismus für Kant in diesem Kontext nur sehr begrenzt angemessen zu sein scheint). Ich würde mir wünschen, dass die gegenwärtige Philosophie des Geistes interessante Interpretationen von Kant und Husserl ernsthaft diskutieren würde. ${ }^{3}$ Und gerade weil dies wünschenswert ist, wäre es gut gewesen, wenn diese Herausforderung weniger polemisch vorgebracht würde und Streubel sich mit dem kritisierten Diskurs auseinandergesetzt hätte, statt ihn pauschal anzufallen.

Noch problematischer sind die Stellungnahmen zur Ethik. War zu Beginn des Buches das gesamte Projekt noch mit einer Grundlegung der Ethik (19) begründet worden, so finden sich im Kapitel „Geist“ nur ein paar lapidare Bemerkungen zur Diskreditierung der Ethik: Es fehlt „die eine Ethik, die uns sicher im Leben und Handeln orientiert. Ob es sie jemals geben wird, ist fraglich“ (329). Diese schlichten Bemerkungen werden mit ein paar abfälligen Aussagen zum derzeitigen Diskurs garniert. Vielleicht gibt es ja irgendwelche Gründe, an der Möglichkeit der Ethik zu zweifeln, in diesem Buch werden sie jedenfalls nicht vorgetragen.

3 Den besten Ansatz dazu böte m. E. eine Diskussion von B. Longuenesse, I, Me, Mine. Back to Kant, and Back Again, Oxford 2017. 
Dass Streubel sich jedoch nicht die Mühe macht, zumindest einmal zu untersuchen, welche Fragen hinsichtlich der Konsequenzen für die Ethik man denn im Anschluss an die skizzierte Konzeption von Leib und Körper stellen müsste, wirft Zweifel daran auf, ob solche Konsequenzen überhaupt im Blick gewesen sind. Um keine Missverständnisse aufkommen zu lassen: Es geht nicht darum, dass in diesem Buch keine Ethik ausgearbeitet wird - eine solche Beschränkung ist vollständig legitim. Aber es geht darum, dass überhaupt kein Versuch unternommen wird, die Frage nach den Konsequenzen für die Ethik seriös zu thematisieren jenseits vorurteilsbehafteter Polemik.

Mit den Undeutlichkeiten hinsichtlich Natur- und Moralphilosophie stellt sich aber die Frage, wie man denn überhaupt von den skizzierten Überlegungen zu einer Anthropologie gelangen könnte. Jedenfalls gibt es eine beträchtliche Diskrepanz zwischen dem Anspruch, mit dem Streubel die Notwendigkeit der Husserl'schen Letztbegründung für ein umfassendes Verständnis vom Menschen begründet, also der Bedeutung von Husserls Konzept für die vierte, alle Fragen umfassende kantische Frage „Was ist der Mensch?“, und dem Unwillen des Autors, sich mit den Fragen danach, was wir naturwissenschaftlich wissen können und was wir tun sollen, überhaupt seriös auseinanderzusetzen. Die kantische Exposition der Frage „Was ist der Mensch?“ würde mehr Ernsthaftigkeit verdienen, und der Weg zu einer Husserl'schen philosophischen Anthropologie, die als strenge Wissenschaft wird auftreten können, ist ein durchaus vielversprechender Weg, aber er ist leider nach Lektüre des Buches noch nicht in Sicht. Dieser Mangel an Ernsthaftigkeit ist bedauerlich, denn als systematische Explikation des Husserl'schen Programms ist dieses Buch in jeder Hinsicht sehr lesenswert. 